\title{
Imaging in oncology - from unknown primary to therapy response assessment
}

\section{Lawrence $\mathrm{H}$ Schwartz MD}

\section{Imaging the unknown primary}

The management from both a clinical and radiological perspective of patients who present with metastases of unknown primary origin is a clinical challenge, despite an increasing number of imaging modalities available to image these patients. The importance of correctly identifying the site of primary tumor relates to the therapeutic approach to these patients and the differences in survival based upon finding and treating the primary tumor.

Unknown primary tumors actually encompass a heterogeneous group of tumors with various clinical presentations, imaging features, treatments and outcomes. One of the most common presentations of unknown primary is in the enlargement of cervical lymph nodes. Other common presentations include axillary adenopathy, ascites, bone or marrow metastases. Because of the heterogeneous nature of this tumor, some have advocated whole body imaging tech- niques, while others have advocated focused imaging based upon the site of initial suspicion and any other clinical information which may help the radiologist differentiate sites of tumors.

In patients that present with enlargement of cervical lymph nodes, palpation and localization may be helpful in determining the site of the primary tumor. Additional sonographic guided needle aspiration may provide additional information. Specifically, the type of malignancy; squamous, undifferentiated or adenocarcinoma may be determined which will help define the site of primary tumor. In approximately $12 \%$ of patients, the primary tumor site cannot be located in patients who present with cervical lymphadenopathy. CT and MRI play a role in evaluation of the neck in all these patients, increasingly, however whole body imaging is being used. Initial studies suggest that FDG PET scanning for the detection of unknown primary cancer may be helpful. In one study, PET revealed pathologic accumulations of FDG in 27 of 53 patients.

Another subset of patients with unknown primary cancers present with axillary lymph node metastases. MRI may prove to be the imaging modality of choice in patients presenting with axillary adenopathy positive for adenocarcinoma but negative physical examination and negative mammogram. Although treatment is controversial, most patients undergo mastectomy. In a study at MSKCC 16 patients who presented with biopsy proven adenocarcinoma to the axilla were evaluated with breast MRI. The primary breast cancer was identified on MRI in $12 / 15(80 \%)$. There was one false positive and no false negative examinations. With knowledge of the MRI results, 11 patients underwent ultrasound and $9 / 11$ (82\%) were identified on ultrasound. Whether these lesions would have been found on ultrasound without knowledge of the MRI results is uncertain. As ultrasound is more widely available and less costly than MRI, a comparison of these two modalities would warrant further study.

Breast MRI performed for breast cancer detection requires the use of a contrast agent and relies on the inherent vascularity of malignant breast lesions. The problem is that benign lesions can also be vascular and demonstrate enhancement. Therefore, false positives can pose a significant problem in interpretation of the exam. Fibroadenomas, recent scars, proliferative and non-proliferative fibrocystic changes, inflammatory processes, atypia, radial scar, and LCIS can all demonstrate enhancement.

\section{Imaging in therapy response assessment}

The response of tumors to therapeutic agents such as chemotherapy and radiotherapy is commonly assessed on radiologic images. 
Radiologic images provide critical information about changes in tumor size on serial examinations performed prior to, during, and after chemotherapy or radiation therapy regimens. Such an assessment cannot reliably be obtained from physical examination in most cases, yet is essential for determining whether or not the particular therapy is benefiting the patient, or whether a particular experimental therapeutic agent is effective against a specific tumor.

The remainder of this talk will be to review techniques using conventional and novel imaging modalities to assess therapy response, including computed tomography (CT), magnetic resonance imaging (MRI) and positron emission tomography (PET). Clinical questions such as which modality to use, how many lesions to measure, and what technique to measure will be addressed. Comparison will be made between currently used criteria for therapy response and the value of newer techniques including PET and MRI perfusion kinetics will be discussed.

The World Health Organization (WHO) response assessment criteria was set up in 1979 to standardize the recording and reporting of response assessment for solid tumors so that the response outcomes can be compared between various research organizations, trials, and therapies. Even though a tumor is three dimensional, the response assessment is performed on the basis of measurements from cross sectional scans in two dimensions. After therapy, the percentage reduction or percentage increase in the corresponding measurements is used for calculating response assessment. WHO criteria recommended the change in the cross product as the method for evaluating therapy response.

Several changes in the WHO criterion have been recognized over time resulting in situations where responses are no longer comparable. The various sources of variabilities have been in the definition of 'measurable' and 'evaluable' lesion, the minimum lesion size and the number of lesions to be recorded for patients with multiple lesions, the definition of progressive disease, and the processing and analysis of imaging data from relatively new technologies such as CT/MRI. Disease progression as defined by a $25 \%$ increase is used by some groups as the increment in a single lesion and the change in the total tumor burden by others.

In 1994, several organizations, such as the European Organization for Research and Treatment of Cancer, Belgium, National Cancer Institute, USA, and others started to review these issues with the intent of revising the WHO criterion based on the experience and knowledge accumulated since its initiation. Under these principles, Response Evaluation Criteria in Solid Tumors (RECIST) guidelines have been published. Three primary changes made were 1) adopting uni-dimensional measurement (in terms of a tumor's maximum diameter) as the underlying metric for response assessment, 2) making the cutoff point for definition of progressive disease higher, and 3) specifying very clear cut guidelines about minimum lesion size and the number of lesions to consider for response assessment of a patient with multiple lesions. The minimum lesion size at which a tumor will be considered measurable is decided as $>=20 \mathrm{~mm}$ for conventional imaging modalities
(X-Ray, CT, MRI) and $>=10 \mathrm{~mm}$ for spiral CT scan. These cutoffs are imposed to avoid measurement error. All measurable lesions up to a maximum of 5 per organ and 10 lesions in total, representative of all involved organs are to be used for response assessment.

Response data from several trials were re-analyzed by both criteria to assess the extent of agreement between them. James et al analyzed 569 patients accrued on 8 Phase II and Phase III studies of various cancers and reported a kappa coefficient of 0.95 as a demonstration of excellent agreement between the response and non-response categories as assigned by WHO and RECIST criteria. Only $12 \%$ less patients were found to move to the SD category from the progression category $(n=128)$ due to the stricter RECIST definition of progressive disease.

We have seen greater differences between RECIST and WHO. In a group of 25 patients on clinical trials, there was a significant difference in percentage change in measurement of tumors using uni-dimensional measurement compared to bi-dimensional cross-products. The therapeutic response assessment in 20\% (5/25) of patients would be changed if unidimensional measurements were used and in $8 \%(2 / 25)$ of patients if volumetric measurements were used. There was no statistically significant difference in cross-product or area response assessment, nor would any therapeutic response be reclassified if area calculation was used instead of cross-product.

There are also other methods of assessing therapy response, including the use of PET scanning and functional MRI. These will be discussed, 


\section{STAR ABSTRACT}

compared and contrasted with more standard techniques outlined above.

\section{Suggested reading}

1. Miller $A B$, Hoogstraten $B$, Staquet $M$, et al. Reporting results of cancer treatment. Cancer 1981; 47: 207-214.

2. Therasse P, Arbuck SG, Eisenhauer EA, et al. New guidelines to evaluate the response to treatment in solid tumors. J Natl Cancer Inst 2000; 92: 205-216.

3. James K, Eisenhauer E, Christian M, et al. Measuring response in solid tumors: Unidimensional versus bidimensional measurement. J Natl Cancer Inst 1999; 91: 523-528.

4. Dachman AH, MacEneaney PM, Adedipe A, et al. Tumor size on computed tomography scans: Is one measurement enough? Cancer 2001; 91: 555-560.

5. Schwartz LH, Ginsberg MS, DeCorato D, et al. Evaluation of tumor measurements in oncology: Use of film-based and electronic techniques. Clin Onc 2000; 18(10): 2179-2184.

6. EA Morris, LH Schwartz, MB Drotman, SJ Kim, LK Tan, L Liberman, AF Abramson, KJ Van Zee, and DD Dershaw. Evaluation of pectoralis major muscle in patients with posterior breast tumors on breast MR images: early experience. Radiology 2000; 214: 67.

7. EA Morris, LH Schwartz, DD Dershaw, KI Van Zee, AF Abramson, and I Liberman. MR imaging of the breast in patients with occult primary breast carcinoma. Radiology 1997; 205: 437.

8. KH Bohuslavizki, S Klutmann, and S Kröger. FDG PET detection of unknown primary tumors. J Nucl Med 2000; 41: 816 .

call Corinth, your fast track to the UK 0800201120 E-mail corinth@icon.co.za

Radiographers

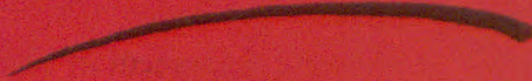

Audiologists Biomedical Scientists Cardiographers CSO/Cardiac Technicians Dental Nurses

Dieticians Doctors/Specialists

Medical Admin and Clerical

Medical Laboratory Technicians

Medical Secretaries Occupational Therapists Psychiatrists Physiotherapists Radiographers Social Workers Speech and Language Therapists Theatre Nurses 